El sujeto humano son dos y no uno: notas sobre el feminismo de la diferencia

Alejandro Alfredo Rafael Silva Fernández

http://perio.unlp.edu.ar/ojs/index.php/question/article/view/4445

Cita sugerida: Silva Fernández, A. (2018). El sujeto humano son dos y no uno: notas sobre el feminismo de la

diferencia. Question, 1(58), e050. doi:https://doi.org/10.24215/16696581e050

\title{
El sujeto humano son dos y no uno: notas sobre el feminismo de la diferencia
}

\author{
The human subject are two and not one: notes about the difference \\ feminism
}

Alejandro Alfredo Rafael Silva Fernández silvaferale@gmail.com

http://orcid.org/0000-0003-4054-2608

Instituto de Investigaciones Geohistóricas;

Universidad Nacional del Nordeste/

Consejo Nacional de Investigaciones Científicas y Técnicas (Argentina)

\section{Resumen}

La teoría de la diferencia sexual tiene su origen y anclaje en Europa y centra su análisis en el sexo, entendido como dato no accidental, auténtico, que ha quedado impensado e irrepresentado en el dominio del orden simbólico masculino. En este contexto, apunta a reivindicar la diferencia a partir de la situacionalidad de las mujeres y las potencialidades que acarrea la subjetividad en su capacidad de acción política. El objetivo de este trabajo es dar cuenta de los debates que toman como punto de problematización a la diferencia sexual e identificar los puntos de dialogo y sus potencialidades de vigencia en el contexto contemporáneo en los desarrollos de Rosi Braidotti y los trabajos que María Luisa Femenías comparte con María Marta Herrera y María de los Ángeles Ruiz como nociones superados de esencialismos. 
Palabras clave: Feminismo; diferencia sexual; identidades; sujetos.

\section{Abstract}

The theory of sexual difference has its origin and anchorage in Europe and focuses its analysis on sex, understood as non-accidental, authentic data that has been unthinkable and unrepresented in the domain of the masculine symbolic order. In this context, it points to claim the difference from the situatedness of women and the potential it brings subjectivity in its capacity for political action. The aim of this paper is to report the discussions that take as their point of problematización sexual difference and identify the points of dialogue and its potential effect in the contemporary context in the developments of Rosi Braidotti and jobs that María Luisa Femenías shares María Marta Herrera and Maria de los Angeles Ruiz as overcome notions of essentialism.

Keywords: Feminism; sexual difference; identities; subjects.

Partiendo de la premisa de que lo contrario de la igualdad no es la diferencia, sino la desigualdad; y que la reivindicación de las diferencias no invalida ni contradice la reivindicación de igualdad, el feminismo de la diferencia sexual apunta a reivindicar la diferencia a partir de la situacionalidad de las mujeres y las potencialidades que acarrea la subjetividad en su capacidad de acción política. Este debate devuelve a la escena una pregunta que es central al hacer referencia a sujetos silenciados, en este caso acerca del sujeto del feminismo con el objetivo de huir de los esencialismos excluyentes sin olvidar las diferencias y desigualdades entre mujeres y varones y también entre mujeres y sus condiciones de vida.

La teoría de la diferencia sexual tiene su origen y anclaje en Europa, mientras que la teoría de género es de tradición anglo norteamericana. La primera centra su análisis en el sexo, entendido como dato no accidental, auténtico, que ha quedado impensado, irrepresentado en el dominio del orden simbólico masculino. El cual es responsable de imponer la lógica de lo mismo -lo uno, el ser, el hombre- y lo otro -lo negado, lo subordinado, la mujer-. La diferencia sexual analiza la asimetría de la relación entre los sexos, y también la separación de la esfera de lo simbólico y de lo social, postulando que lo simbólico, precede y organiza lo social. Existe aquí una diferencia con los estudios de género que, en general, muestran inclinación hacia el 
sociologismo, no teniendo en cuenta la dimensión simbólica y psicoanalítica por la que se establece en el lenguaje lo femenino y lo masculino (Burgos Díaz, 2005).

Este trabajo tomará como referencia tres textos que problematizan y debaten acerca de la irrupción de la diferencia, el rol del cuerpo sexuado frente a las concepciones que lo mantienen relegado y la noción de sujetos nómades como instancia superadora de los esencialismos. Para esto se referenciarán los desarrollos de Rosi Braidotti (2004) en Feminismo, Diferencia Sexual y Subjetividad Nómade; María Luisa Femenías y María Marta Herrera (2008) en "Los Derroteros de la Diferencia" y de Femenías y María de los Ángeles Ruiz (2004) en "Rosi Braidotti, diferencia sexual y condición nómade". El objetivo estará centrado en dar cuenta de los debates que toman como punto de problematización la diferencia sexual e identificar los puntos de diálogo y sus potencialidades de vigencia en el contexto contemporáneo.

\section{El sujeto del feminismo}

En el primer capítulo de su libro Feminismo, Diferencia Sexual y Subjetividad Nómade, Rosi Braidotti (1999) problematiza al sujeto del feminismo partiendo de algunas escenas que dan cuenta de las posibilidades, o acaso imposibilidades, del acceso de las mujeres a la educación superior, para luego avanzar hacia las implicancias de la categoría de mujer en el feminismo. Pese a las diferencias que existen en la concepción individual de las mujeres, se identifica a la mujer como una categoría signada por supuestos comunes culturalmente impuestos. Entre esos, cuestiona la autora que todas las mujeres se hallan excluidas de las mismas condiciones de posibilidad formativa a las que pueden acceder los varones.

Frente al estado de situación anteriormente planteado, Braidotti encuentra una relación causal con la idea preestablecida de mujer que posee la cultura occidental, cuya consecuencia es la escisión de todas las mujeres de los derechos a la educación porque en su representación tradicional es considerada como irracional, hipersensible, destinada a ser esposa y madre, cuerpo, sexo y pecado. Pero fundamentalmente, la mujer es posicionada como "distinta de" el hombre. Esta diferencia impuesta posee, entre otras dimensiones, una relación causal con la misoginia, a la que la autora define y caracteriza, retomando a Virginia Woolf, no como un acto irracional de odio a la mujer sino como una necesidad estructural y un paso lógico en el proceso de construir la identidad masculina en oposición y rechazo a la mujer, vinculándola al patriarcado por la negación. 
La paradoja de ser definida por otros reside en que las mujeres terminan por ser definidas como otros: son representadas como diferentes del Hombre y a esta diferencia se le da un valor negativo. La diferencia es, pues, una marca de inferioridad (Braidotti, 2004: 13).

En este contexto, el estándar normal se posiciona en lo masculino y encuentra su reafirmación en la descalificación del sujeto femenino como una necesidad estructural en un sistema que construye las diferencias como oposiciones.

Para Braidotti, el momento fundacional de la historia feminista es la afirmación de un lazo entre todas las mujeres, de una relación entre ellas, que existe en la medida en que comparten la misma categoría de diferencia entendida como negativa. Retoma el posicionamiento de De Beauvoir en su aclaración acerca de que ella no podía pensar adecuadamente en su propia existencia individual sin tomar en cuenta la condición general de las mujeres y la categoría de mujer, como construcción patriarcal, sentando las bases para una nueva clase de sujeto femenino: una categoría política y teórica "sujeta al cambio" en términos de Nancy Miller o un "sujeto feminista femenino" en palabras de Teresa de Lauretis.

La pensadora feminista femenina toma como objeto de estudio la experiencia de las mujeres y la categoría de Mujer, y lo hace no sólo para comprender el mecanismo de descalificación de su género, sino también para liberar a la noción de Mujer de la red de semi verdades y prejuicios adonde la confinó el patriarcado ((Braidotti, 2004: 14-15).

La experiencia de las mujeres en la vida real será la noción central que sustenta este proyecto. Adrienne Rich expresa esta idea en la noción de "política de localización", entendida en tanto el pensamiento y el proceso teórico no son abstractos, universalizados, objetivos ni indiferentes, sino que están situados en la contingencia de la propia experiencia donde la posicionalidad resulta clave.

Esta posicionalidad del marco conceptual feminista encuentra su sitio primario de localización en el cuerpo.

El sujeto no es una entidad abstracta sino material incardinada o corporizada. El cuerpo no es una cosa natural; por el contrario, es una entidad socializada, codificada culturalmente; lejos de ser una noción esencialista, constituye el sitio de intersección de lo biológico, lo social y lo lingüístico, esto es, del lenguaje entendido como el sistema simbólico fundamental de una cultura (Braidotti, 2004: 16). 
Ese contexto cultural establece una dicotomía sexual que situó sistemáticamente a las mujeres en el polo de la diferencia, entendida como inferioridad respecto de los hombres. La pregunta feminista femenina es entonces, de qué manera potenciar la diferencia que la mujer establece para poner en práctica nuevos valores.

El sujeto del feminismo para Braidotti, sigue siendo la mujer como categoría pero no conceptual, sino una mujer que adquiere corporeidad en la que se interceptan lo biológico, lo social y lo lingüístico. Un cuerpo donde batalla el orden simbólico de la cultura en una disputa que históricamente reafirma sus fuerzas descalificando a las mujeres como la alteridad, como lo otro diferente, en una diferencia de connotaciones negativas, no como posibilidades viables de ser potencializadas. La mujer desde un prisma patriarcalizado no posee capacidades ni posibilidades de ser un sujeto político y es ahí donde la política de localización puja hacia una reconfiguración de la mujer como la diferencia alterizada, silenciada.

\section{Diferencia sexual y condición nómade}

En el texto "Rosi Braidotti, diferencia sexual y condición nómade" Femenías y Ruiz (2004) toman como punto de partida, en su reflexión acerca de los desarrollos de Braidotti sobre el feminismo de la diferencia y el carácter articulador del nomadismo, al episodio dado a comienzos de los sesenta en Francia, en torno a un conjunto disonante de voces que anunciaron un nuevo orden filosófico. El mismo estaría basado en la relectura crítica de la dialéctica de Hegel a partir de las influencias de Heidegger y del estructuralismo de LéviStrauss. Este proyecto posibilitó la apertura de una concepción de la "diferencia", ya no en términos de contradicción sino como diferencia originaria, a la que Jacques Derrida denominó différance, que se vincula con el verbo "diferir", es decir, "no ser idéntico" o como equivalente a "posponer", "dejar para más adelante". En 1970, aunque no logra escapar a la impronta hegeliana, Michel Foucault en su lección inaugural en el College de France defendió también la idea de una "huida" o "fuga" de ese reino a los efectos de construir otro modo de filosofar.

Para Femenías y Ruiz este giro provocó una nueva concepción de la historia, del origen, de la representación y de la razón, que siguen siendo, como denuncia Luce Irigaray en Speculum, falogocéntricos. Mantienen un orden simbólico fálico en el cual la nueva filosofía mantiene una deuda en poner al descubierto la diferencia más radical, la otredad más absoluta: la diferencia de los sexos entendida como el principio de inteligibilidad de la cultura occidental. 
El punto central que hace del trabajo crítico de Braidotti una reflexión sumamente innovadora, consiste en denunciar la complicidad entre racionalidad y masculinidad:

Toda teoría del sujeto se ha adecuado siempre a lo masculino. Sometiéndose a ello, la mujer renuncia sin saberlo a la especificidad de su relación con lo imaginario. Y se coloca una vez más en situación de ser objetivada por el discurso en cuanto femenino. En él se reobjetiva a sí misma cuando pretende identificarse como un sujeto masculino (Femenías y Ruiz, 2004: 2).

Para el feminismo de la diferencia sexual, el denominado feminismo de la igualdad sólo entiende lo otro (mujer) en términos de la lógica de lo mismo, de la homologación al modelo normativo del varón, posición de la que Braidotti intenta apartarse.

La autora apela al concepto de glo-calización para dar cuenta de la tensión actual entre lo local y lo global y los efectos de esa tensión irreversible, donde el borramiento de fronteras nacionales atenta contra las identidades de los sujetos. En este contexto, el término nomadismo resulta central en la mayor parte de sus argumentaciones y constituye una práctica articuladora que subraya el hecho de que los sujetos están en tránsito, pero al mismo tiempo, los suficientemente anclados en una posición histórica como para aceptar la responsabilidad que les cabe en ella (Amado, 1999; Femenías y Ruiz, 2004).

La figuración del nómade constituye una forma de intervención en el marco del debate entre el feminismo y la crisis que trae aparejada la posmodernidad, que se pone de manifiesto en el desafío a los valores y las representaciones tradicionales del sujeto. En el nomadismo activo, lo político no es más que la toma activa de conciencia de la fragmentación como característica propia del sujeto y por ende el abandono de una visión sustantiva del sujeto. El punto de partida de Braidotti es la búsqueda incesante de nuevas posibilidades que, a su vez, no hagan posible la constitución de hegemonías totalizadoras. "De ahí que para nuestra autora el feminismo como movimiento muestra la ruptura crítica del falso universalismo y del sujeto monolítico al tiempo que afirma rotundamente el deseo de las mujeres de legitimar múltiples formas de subjetividad" (Femenías y Ruiz, 2004: 4).

En su obra Patterns of Dissonance (1991) Braidotti identifica que el desafío de la sexuación no se resuelve negándola sino examinando y poniendo el acento en la naturaleza corpórea del sujeto sexuado, lo cual configura una deuda de la filosofía francesa. En efecto, a diferencia de Judith Butler que niega el sustrato biológico en pos de un hiperconstructivismo, el asunto de la sexuación y de la incardinación del sujeto forma parte de una posición anti-cartesiana. 
En Sujetos Nómades (1994), la autora se propone cartografiar una teoría materialista y transmóvil de la subjetividad feminista, que trabaje dentro de los parámetros de los predicamentos postmodernos, sin romantizarlos pero sin nostalgia por las totalidades del pasado. Para ello es preciso desarrollar una concepción de la subjetividad nómade, suspendiendo los sistemas binarios de conceptualización y los hábitos monológicos del falocentrismo a fin de generar una conciencia nómade como imperativo político para alcanzar el pensamiento crítico. Se llega a ser sujeto a través de una serie de interdicciones y permisos, que inscriben la propia subjetividad en la base del poder, como un conjunto de partes fragmentadas que se mantienen unidas gracias al adhesivo simbólico que es el apego al orden falogocéntrico o a su identificación con él. Quebrado ese orden, el sujeto deviene nómade.

La diferencia sexual debe llevar a reconsiderar discursos y prácticas de la diferencia a fin de relevar connotaciones falogocéntricas como práctica política crítica de los supuestos del feminismo igualitarista y deshacer los discursos éticos y políticos tradicionales sobre la otredad. Lo "otro" marginal (las mujeres) tiene sentido en relación con un "centro" masculino. Cuestionar el centro como hacen las feministas supone cuestionar también la propia inscripción.

\begin{abstract}
De esto Braidotti concluye que las políticas feministas desafían las estructuras de representación y los valores sociopolíticos atribuidos a La Mujer como lo Otro en un sistema patriarcal. Pero, al mismo tiempo, cuando eligen la posición feminista se constituyen en sujetos fragmentados y no entidades racionales monolíticas. Braidotti considera a las mujeres, como una multiplicidad en sí mismas, que han abandonado su inscripción tradicional. Marcadas por un conjunto de diferentes inscripciones, fragmentadas, anudadas, se construyen sobre intersecciones de diversos niveles de experiencia. De ese modo, el sujeto feminista es una unidad volitiva, auto-representacional e inconsciente. En tanto que tal, abriga un conjunto de relaciones mediadas de las estructuras que condicionan su situación de vida (Femenías y Ruiz, 2004: 11).
\end{abstract}

La autora prefiere la noción de "diferencia sexual" a la de "género" y por ende considera que el objetivo del feminismo no debe ser negar la diferencia; hacerlo confirmaría la lógica falocéntrica que solo reconoce la igualdad en términos masculinos, sino que debe reestablecer lo femenino dentro de la diferencia sexual con valor propio para generar un imaginario autónomo más allá de los modelos o estereotipos de varones y mujeres. En su intento de conceptualizar la diferencia como "otro positivo", fuertemente criticado por el feminismo postcolonial,

Braidotti choca con la dificultad de que el signo en el modelo postestructuralista existe sólo en relación diferencial con otros signos. En otras palabras -como muy bien advierte Felski- 
es difícil ver cómo la feminidad autodesignada se reconcilia con un modelo semiótico que define el significado como fundamentalmente relacional, inestable e impuro (Femenías y

Ruiz, 2004: 13).

A partir la búsqueda de otras formas de hacer filosofía en términos de diferencia, de no ser idéntico y aún más radicalmente de huir, en vistas de quebrar formas tradicionales de pensar, Braidotti irá ampliando los márgenes para la emergencia de ideas viables para pensar la situacionalidad de las mujeres. Apartándose del feminismo de la igualdad, que contempla a la alteridad en su potencialidad de homologarse a los parámetros establecidos desde el falogocentrismo, apunta a reivindicar la diferencia, su fragmentación y las múltiples formas de la subjetividad en su capacidad de acción política a contracorriente de los esencialismos, del falso universalismo.

\section{Los derroteros de la diferencia}

En su texto "Los Derroteros de la Diferencia", Femenías y Herrera (2008) avanzan en el desarrollo teórico de la crítica radical al paradigma igualitarista de la modernidad de mediados de los sesenta, reemplazándolo por la noción de reconocimiento de la diferencia, donde contrariamente al modelo anterior- la diferencia es entendida como positiva. Esta será la lógica de la producción teórica "post": postmoderna, postestructuralista, postfeminista donde las autoras se centrarán en el sentido autoafirmativo y positivo que insiste en el carácter distintivo y que remite a aquello que se considera propio o específico en articulación con especificidad étnica -o de raza-, cultural, de clase o de sexo.

En este análisis existe un principio aclaratorio que delimita muchas de las reflexiones acerca de los feminismos de la igualdad y de diferencia: la diferencia no es lo opuesto a la igualdad, sino más bien a la desigualdad y de manera similar su antónimo no es la igualdad sino la identidad. De modo que un feminismo basado en la diferencia como "positivamente otra", por decirlo en palabras de Irigaray, rechaza la lógica de la identidad porque subsumiría a las mujeres a las normas definidas por el varón. Sin embargo no rechaza de plano la igualdad sino que defiende una comprensión materialmente más extendida de la misma.

Quienes defienden la interpretación anteriormente expuesta, entienden a la diferencia sexual como fundante de toda diferencia y por ende prefieren hablar de pensamientos y prácticas políticas basadas en la diferencia sexual más que en las diferencias de género. Consideran que la diferencia sexual es el principio de inteligibilidad de la razón, la posibilidad de 
simbolización, de producción de referencia y de sentido de un tipo particular de conocimiento a partir de un cuerpo particularmente sexuado. El punto de partida es entender que el sujeto humano es dos y no uno, y no en una relación de subordinación, sino de paridad, se sostiene que existe otro logos que el lenguaje patriarcal oscurece, reduce, invisibilidad o niega y que es necesario recuperar en su condición de orden simbólico no falogocéntrico.

El pensamiento de la diferencia sexual intenta pensar al ser humano según una concepción dual donde tanto ser-varón como ser-mujer sean formas primarias. En la cultura occidental, los discursos y los saberes no dan cuenta de que la diferencia sexual afecta a los sujetos de esos discursos y de esos saberes, tanto o más que su ubicación espacio temporal, su finitud. Que ser varón o ser mujer permanezca en la intimidad, sin palabras, sin representaciones, sin lenguaje propio, opera como el mecanismo de negación más acabado: ni siquiera puede ser dicho. Para trabajar en torno a lo anterior, Femenías y Herrera (2008) retoman los postulados de Irigaray, Muraro y Collín como referentes del feminismo europeo de la diferencia sexual.

Luce Irigaray: la circularidad de ser mujer. Iniciadora de esta línea de pensamiento, sostiene que la diferencia sexual es el problema fundamental a resolver en el siglo $\mathrm{XX}$, porque constituye el horizonte de comprensión del mundo. A partir de una severa crítica a la filosofía, la modernidad y el feminismo que se deriva de ella, denuncia que el pensamiento de la diferencia promovido por sus contemporáneos ignora u olvida la más significativa de las diferencias, la diferencia sexual que en la misma línea que Adrienne Rich considera que es el principio de Inteligibilidad de la Cultura Occidental.

En su obra Speculum, Irigaray defiende la tesis de que la filosofía occidental es el edificio más acabado de encubrimiento de la verdad originaria elemental de que todas/os somos nacidos de una mujer. La autora reivindica la corporalidad de las mujeres, en su naturaleza y en su verdad que, en tanto la palabra mediadora es siempre, registro y límite de un orden simbólico masculino. Desde el espacio político esto significa reconocer que las mujeres muy pocas veces, por no decir nunca, piensan o hablan por sí mismas, puesto que todas las mediaciones simbólicas son operativamente masculinas.

La intención de Irigaray "es romper la naturaleza de la explicación causal, romper la seguridad del sujeto lingüístico, desquiciar al lenguaje en su apropiación de la realidad, dejar que el inconsciente aflore con el fin de construir su propio lenguaje", a lo que las autoras se preguntan: ¿si la diferencia silenciada hablara -como quiere Irigaray- la entenderíamos? ¿O cómo entenderla en tanto obedecería a códigos y categorías inconmensurables con las patriarcales ya existentes? (Femenías y Herrera, 2008: 65). 
Luisa Muraro: madre real madre simbólica. La interpretación de la historia de la filosofía occidental y de la cultura patriarcal sostuvo un esquema de rivalidad, de dualidad, entre el mundo ideal de lo verdadero y el mundo de lo real, engañoso e injusto. Muraro advierte que nunca podría alcanzar su independencia simbólica porque estaba inmersa en lo que denominó un "desorden simbólico". Ese desorden consiste en negar lo real y lo material. Lo más importante no es el rechazo a la cultura y a la filosofía patriarcal que se encargaron de la remoción de la relación con la madre, sino desprenderse o des-aprender algo que en verdad nunca tendría que haberse aprendido (u olvidado): "que somos nacidos de mujer". Identificar su convivencia en una cultura en la que no se enseña a las mujeres a amar a la madre le permitió identificar el estado de necesidad en el que se encontraba, en el que la independencia simbólica no se logra a través de la filosofía sino a partir de la política de las mujeres.

La autora explica que, tanto la lengua que hablamos como el mismo saber hablar son el fruto de un acuerdo con lo real que se contrata con la madre, intercambiando reconocimiento de autoridad con facultad del lenguaje. En la vida adulta las mujeres pierden autoridad y competencia simbólica, lo que las lleva a una retórica de la incertidumbre a tratar de decir no lo que es, sino lo que otros dicen o han dicho o dirán que es. La incompetencia simbólica que caracteriza a las mujeres las sumerge en el nihilismo del ser fingido. Así Muraro acuña las nociones de madre simbólica y autoridad materna.

La madre simbólica es la que garantiza la existencia como sujetos, no totalmente separados de la sociedad masculina, pero sin embargo autónomos de la definición y la dominación masculina. Consiste en la alianza entre la mujer que desea y la mujer que sabe, saberse diferentes al varón no les basta a las mujeres para saberse. En el sistema de las relaciones sociales falta una estructura simbólica adecuada a ellas. Esa estructura no debe ser solamente simbólica sino también material/natural: "el continuo materno". Es la estructura simbólica y natural a la que pertenece la hija y que permite expresar y ejercitar la potencia materna, habitando a su vez la posibilidad de sostener una genealogía femenina.

Muraro considera que el orden simbólico actual se puede cambiar y que tal revolución simbólica es política, lo cual llevaría a una auténtica libertad femenina. Esta libertad no consistiría ni exigiría el reconocimiento de los derechos de la mujer, ni de la igualdad bajo la ley patriarcal, sino una respuesta total, política y personal de/para las mujeres, poniendo la responsabilidad del cambio en las mujeres y hacia las mujeres quienes deben tomar posición frente a la comunidad simbólica que produce una genealogía inventada para construir, a partir de prácticas feministas de referencias a las madres simbólicas, un nuevo orden real. 
Francoise Collin: una herencia sin testamento. Collin se pregunta en qué condiciones es posible pensar un mundo común descontando que hay posibilidades de orden simbólico común entre varones y mujeres, el problema estriba en tratar de superar las relaciones de poder entre las categorías de varón y mujer, así como en cuestionar la forma de organización social dualizada y jerarquizada que atraviesa todas las culturas y la historia. La cuestión de los sexos emerge en el pensamiento de muchos filósofos pero de forma accesoria a algún otro problema. En primer lugar la pregunta por el sexo siempre está referida a las mujeres y no se centra en la relación entre los sexos, nunca se cuestiona la posición del sujeto masculino que, en definitiva, es el que filosofa. La diferencia de los sexos no debe considerarse desde una postura esencialista, ni en tercera persona, sino que toda reflexión sobre ella se pone en marcha a partir de la reflexión efectiva y real de mujeres y varones. Admite el carácter social de la construcción de los sexos, dicha construcción no se produce a partir de un modelo natural de ellos, sino que está basado en una metafísica de la acción atravesada por el deseo y guiada por el pensamiento en un mundo plural. La revolución de las mujeres debe partir de un contexto concreto y complejo en el cual, para quebrar con lo establecido, se debe reflexionar desde una confrontación dialógica, para luchar contra la alienación a partir de la cual la lógica de la dominación sustituye el diálogo por el discurso sobre el otro, convirtiendo a ese otro en un objeto del que se habla.

Para Collin es preciso pensar abandonando la pretensión de que una única causa explica todas las injusticias y desigualdades humanas y que existe una concepción de acción y compromiso político anclado en una "praxis de la diferencia sexual", la cual tiene un sentido transformador, es un acto de desplazamiento fuera de los lugares tradicionales, sin que le sea asignado un buen lugar. La diferencia entre los sexos no se puede anular bajo el supuesto de que es una construcción social, ni estancarse en esencialismos eternos e inamovibles. Se trata más bien de desplazarse, de recrearse en la pluralidad de lo social a través del lenguaje.

Tomando como punto de partida que el sujeto humano es dos y no uno, Femenías y Herrera (2008) avanzan en el debate acerca de la postura asumida por estas autoras y el énfasis puesto en la diferencia sexual más que en las diferencias de género, ya que, según lo plantean las autoras referenciadas, se trata del principio de inteligibilidad de la razón. Es así que Irigaray reivindica la corporalidad de las mujeres, en su naturaleza y en su verdad, en tanto la palabra mediadora es siempre registro y límite de un orden simbólico masculino. Muraro sobre el desorden simbólico en el que la cultura se encargó de invisibilizar a la madre y que debe ser 
modificado en vistas a construir un nuevo orden real a partir de prácticas que depositen la responsabilidad de cambio en las mujeres y Collin acerca de que la diferencia de los sexos no debe considerarse desde una postura esencialista, ni en tercera persona, sino que toda reflexión sobre ella se pone en marcha a partir de la reflexión efectiva y real de mujeres y varones.

\section{A modo de conclusión}

La estratagema cultural de posicionar a la mujer como "distinta de" el hombre en términos negativos, es identificada en los desarrollos expuestos como una necesidad estructural y un paso lógico en el proceso de construir la identidad masculina. Además, mientras el proceso de producción de pensamiento y conocimiento, y por ende su circulación, se encuentra reservada al hombre, es el mismo el encargado de definir a la mujer como categoría. La mujer se encontraba, y en muchos espacios aún se encuentra, definida por otros y por ende asumiendo un lugar y una serie de características impuestas donde el estándar esta dado en lo masculino. Esa diferencia ubicada en lo femenino se construye entonces como negativa, como un rasgo de inferioridad.

La posicionalidad de la mujer y su anclaje corporal resultarán elementos clave en el quiebre de la red de supuestos y semi verdades a las que el patriarcado confina a las mujeres, ya que el cuerpo como entidad socializada y culturalmente codificada constituye el sitio de intersección de lo biológico, lo social y lo lingüístico como el sistema simbólico fundamental de una cultura. El cuerpo y su mediatización se torna entonces un principio de disputa simbólica y de oportunidades de transformación del orden falogocéntrico, que mantiene oculta a la otredad más radical, la diferencia de los sexos entendida como principio de inteligibilidad.

La figuración del sujeto nómade posee, en este contexto, una doble responsabilidad en la reflexión. Entender la dimensión situacional de los sujetos en tanto entidades en tránsito contra los universalismos y los esencialismos, pero un tránsito lo suficientemente anclado histórica y culturalmente como para asumir una responsabilidad política crítica con su situacionalidad actual y los devenires posibles. Aunque resulta una deuda en este quiebre reconocer el alcance y las posibilidades operativas de la generación de otro orden capaz de tensionar al falogocéntrico y revelar otro logos de sentido, teniendo en cuenta que los significados son fundamentalmente relacionales, inestables e impuros. 
La misión está puesta entonces en lograr una posicionalidad que identifique que el sujeto humano es dos y no uno, y no en una relación de subordinación, sino de una paridad que debe resultar de la puesta al descubierto de ese otro logos que el lenguaje patriarcal oscurece, reduce, invisibiliza o niega y que es necesario recuperar en su condición de orden simbólico no falogocéntrico. Donde además, la corporalidad debe traducirse en una concepción dual en la que ser varón y ser mujer sean formas primarias.

Joan Scott sugiere que el enfrentamiento entre igualdad y diferencia es innecesario y que no debe culminar en un estático punto muerto, sino en una paradoja que debe ser pensada dentro del problema del tiempo y del funcionamiento de un modo específico de transformación histórica.

\footnotetext{
En la medida en que actuó por las "mujeres", el feminismo produjo la "diferencia sexual" que procuró eliminar. Esta paradoja -la necesidad tanto de aceptar como de refutar la diferencia sexual- fue condición constitutiva del feminismo como movimiento político a través de su larga historia (Butler, 2011: 33)
}

Entonces esa diferencia en articulación con las ideas con las cuales dialoga, y en algunos casos rechaza, puede ser comprendida en términos de esta paradoja situacional que lejos de estancar posiciones puede ser reapropiada como motor hacia la revelación de aquello que se denuncia invisibilizado, es decir, a la mujer como entidad en su paridad con el hombre pero constituida sobre la base de sus diferencias.

\section{Bibliografía}

Amado, A. y Domínguez, N. (1999). Rosi Braidotti: diferencia sexual y nomadismo. Mora, 5, pp. 4-7.

Braidotti, R. (1991/1996). Patterns of Dissonanace. Oxford: Polity Press.

Braidotti, R. (2000). Sujetos nómades. Buenos Aires: Paidós.

Braidotti, R. (2004). Feminismo, Diferencia Sexual y Subjetividad Nómade. Barcelona: Gedisa.

Burgos Díaz, E. (2005). Conflicto de paradigmas: "género" y "diferencia sexual". Themata. Revista de Filosofía, 35, pp. 713-720.

Butler, J. (2011). Speaking up, talking back: Joan Scott's critical feminism. En Butler, J. y Weed, E. (eds.). The question of gender: Joan W. Scott's critical feminism. Bloomington: Indiana University Press. 
Femenías, M. L. y Herrera, M. M. (2008). Los derroteros de la Diferencia. Revista Maracanan. Dossiê Diferenças e Desigualdades, IV(1), pp. 63-82. Recuperado de http://www.epublicacoes.uerj.br/index.php/maracanan/article/view/13227/10106

Femenías, M. L. y Ruiz, M. A. (2004). Rosi Braidotti, diferencia sexual y condición nómade. Revista de la Escuela de Historia, Universidad Nacional de Salta, 1(3), pp. 63-83. Recuperado de http://www.redalyc.org/pdf/638/63810305.pdf

Irigaray, L. (1974/1978). Speculum. Paris: Les éditions de Minuit.

Scott, J. (2012). Las mujeres y los derechos del hombre. Feminismo y sufragio en Francia 1789-1944. Buenos Aires: Siglo Veintiuno. 